Research Paper

\title{
The Diagnostic and Prognostic Value of Digital Rectal Examination in Gastric Cancer Patients with Peritoneal Metastasis
}

\author{
Jing Guo ${ }^{1,2^{*}}$, Yongming Chen ${ }^{1,2^{*}}$, Zhimin Liu ${ }^{1,2^{*}}$, Yuanxiang Guan ${ }^{1,2}$, Wei Wang1,2, Xiaowei Sun ${ }^{1,2}$, Wei Li1,2, \\ Dazhi $\mathrm{Xu}^{1,2}{ }^{\boxplus}$ \\ 1. State Key Laboratory of Oncology in South China, Collaborative Innovation Center for Cancer Medicine, Guangzhou, China; \\ 2. Department of Gastric Surgery, Sun Yat-sen University Cancer Center, Guangzhou, China. \\ *Equal contributors \\ $\square$ Corresponding author: Dazhi Xu, Department of Gastric Surgery, Sun Yat-sen University Cancer Center, Guangzhou, China, 651\# East Dongfeng road, \\ Guangzhou510060, Guangdong Province, China. Fax: (+86) 020-87343737. Email: xudzh@sysucc.org.cn \\ (c) Ivyspring International Publisher. This is an open access article distributed under the terms of the Creative Commons Attribution (CC BY-NC) license \\ (https://creativecommons.org/licenses/by-nc/4.0/). See http://ivyspring.com/terms for full terms and conditions.
}

Received: 2018.09.10; Accepted: 2019.01.09; Published: 2019.02.23

\begin{abstract}
Background: Peritoneal metastasis (PM) is the most common cause of death in gastric cancer (GC) patients. However, diagnosis of PM is still difficult in clinical practice. This study aimed to explore the diagnostic and prognostic value of digital rectal examination (DRE) in GC.

Methods: 247 GC patients with PM confirmed by operation were included. The diagnostic yield of DRE compared with computed tomography (CT) was calculated. In another group of 1330 cases receiving radical gastrectomy, 38 cases with DRE $(+)$ postoperatively were analyzed to identify risk factors. A nomogram was constructed to predict postoperative DRE (+).

Results: The specificity, positive predictive value and positive likelihood ratio of DRE in diagnosis of PM was $99.8 \%, 91.2 \%$ and 58.4 , higher than CT $(97.6 \%, 64.9 \%$ and 10.4$)$. Though the sensitivity of DRE (12.6\%) was lower than CT (24.7\%), 17 of 31 patients with DRE $(+)$ could not be found by CT. Moreover, the overall survival of confirmed PM patients with DRE $(+)($ PM-DRE $(+))$ was much lower than PM-DRE $(-)$ patients $(P<0.001)$. In addition, the nomogram to predict postoperative DRE $(+)$ had a bootstrap-corrected concordance index of 0.73 and was well calibrated.

Conclusions: GC patients with DRE (+) could be regarded as a special subtype of stage IV ones with poorer prognosis. Supply of palliative care and chemotherapy rather than unnecessary operation might be a better alternative for these patients. DRE was an effective supplement for CT and should be generally recommended for GC patients.
\end{abstract}

Key words: digital rectal examination, peritoneal metastasis, gastric cancer, $\mathrm{CT}$, nomogram

\section{Introduction}

Gastric cancer (GC) is the second most common cause of cancer-related mortality worldwide, especially in many Asian countries[1-3]. Peritoneal metastasis $(\mathrm{PM})$ is the most common cause of death in GC patients. A major problem in treating PM is how to detect metastasis early so as to stage accurately and select appropriate treatment[4].

Currently, laparoscopy is regarded as an effective diagnostic tool for PM, which can supply a chance to biopsy under vision[5-9]. According to a meta-analysis by Ramos et al, the sensitivity and specificity of staging laparoscopy was $84.6 \%$ and $100 \%$ [10]. However, the laparoscopic approach remains underused $(<10 \%)$ due to its invasive procedure, high cost and the possibility of port-site metastasis [10]. In fact, most cases of GC occur in rural areas of developing countries such as China. These areas lack of sophisticated equipment and well- 
trained surgical teams. Laparoscopic accessibility and availability for PM is very limited.

To date, multidetector computed tomography (CT) scans is the first choice for detecting PM of GC as the preoperative modality[6-8]. CT is widely available in most medical centers because of its less invasion and cost compared with laparoscopy. However, the accuracy of CT is low in identifying PM, even with modern CT techniques[11]. In addition, 18F-fluorodeoxyglucose positron emission tomography (18F-FDGPET) is also applied to detect PM. However, 18F-FDG-PET is unreliable due to its high cost and low sensitivity, especially for diffuse-type GC[12].

Digital rectal examination (DRE), as a simple, cheap and noninvasive clinical tool available, has been advocated for prostate and rectal cancer detection[13, 14]. DRE is also a useful approach for the diagnosis of anorectal disorders[15]. As PM often appears as mass or hard nodules in the Douglas' pouch or Rectovesical pouch, DRE is often used as one component of the physical examination for GC patients in many hospitals[16]. However, the diagnostic and prognostic value of DRE in GC patients with PM has not been evaluated yet. We supposed the value of DRE was extremely underestimated in accessing GC. Therefore, we designed this study to compare the effectiveness of DRE and CT for detecting PM in GC patients.

\section{Methods}

\section{Study population and protocol}

1643 GC patients who underwent operation from November 2010 to December 2015 in Sun-Yat Sen University Cancer Center were included in this study. The operations included gastrectomy, bypass gastrojejunostomy and biopsy of metastasis. The overall survival (OS) was calculated from the date of surgery to the date of death or the last follow-up. Median follow-up period was 16 months. The American Joint Committee on Cancer (AJCC) Gastric Cancer Staging version 7 was used for TNM staging[17].

\section{Preoperative clinical assessment}

Preoperative clinical assessment included age, gender, height, weight and DRE. Body mass index $(\mathrm{BMI})=$ weight $(\mathrm{kg}) /($ height $*$ height $) \quad\left(\mathrm{m}^{2}\right)[18]$. Serum tumor markers included CEA, CA19-9 and CA72-4. According to Japanese classification of gastric carcinoma, the degree of peritoneal metastasis is classified as follows: P0, no peritoneal seeding; P1, disseminating metastasis to the region directly adjacent the peritoneum of stomach (above the transversecolon), including the greater omentum; P2, several scattered metastases to the distant peritoneum and ovarian metastasis alone; and P3, numerous metastases to the distant peritoneum[19].

DRE is performed and recorded routinely together with other bedside physical examination in initial interview. At least two experienced surgeons performed DRE and two board-certified radiologists interpreted CT scan for every patient receiving surgery. The surgeons performing the DRE blinded to $\mathrm{CT}$ and laparoscopy results. When the two surgeons were not consistent with each other, we found a senior surgeon to make a final decision. PM often appear as mass or hard nodules in Douglas' pouch or Rectovesical pouch, which can not be moved by finger. Serious PM even appear as "frozen pelvic" and rectal obstruction. The abdominal CT scan was performed using a 64-slice spiral CT (Aquilion TSX-101A, Toshiba Medical System, Tokyo, Japan). A standard imaging protocol of imaging process was adopted. The scanning layer thickness was $5 \mathrm{~mm}$ with a $1-\mathrm{mm}$ pitch, and the scan area included thorax, abdomen and pelvis. Intravenous nonionic contrast material (1.5 ml iopromide per kilogram of body weight, Ultravist 370; Schering, Berlin, Germany) was injected into the antecubital vein at $2.5 \mathrm{ml} / \mathrm{s}$ [20-23]. Ascites was defined as four levels by CT imaging: none $(<100 \mathrm{ml})$, mild $(100-300 \mathrm{ml})$, moderate $(300-1000 \mathrm{ml})$ and massive $(>1000 \mathrm{ml})[24]$.

\section{Statistical analysis}

In our study, histopathologic result was considered as the gold standard for the diagnosis of PM. The $x^{2}$ test was used for categorical variables. The t-test was used for continuous variables. Variables proved statistically significant in the univariate analysis were included into the multivariable logistic regression analysis[25]. Independent risk factors in logistic regression analysis for DRE $(+)$ were assembled into a nomogram. The summary statistic used to evaluate the predictive discrimination of the nomogram was the concordance index (C-index). Possible values of the C-index ranged from 0.5 (random classification) to 1.0 (perfect classification). The calibration curves, which showed the relationship between the predicted and observed risk for each outcome, were generated using 1000 bootstrapping sample. The Kaplan-Meier curve was used for survival analysis.

Statistical analysis was performed by the software statistical package for social sciences version 20.0 (SPSS, Chicago, IL) and R for Windows, version 3.3.3 (http://www.r-project.org/). Nomogram was carried out using the library "rms" in R. P values less than 0.05 were considered as statistically significant. 


\section{Results}

\section{Clinical characteristics of the study population}

A total of 1643 patients underwent operation were enrolled, including 1330 stage I-III and 313 stage IV cases (Figure 1). 247 patients with PM underwent surgery (R1 resection: $n=73, R 2$ resection for obstruction or bleeding: $n=78$, Bypass gastrojejunostomy: $n=44$, Biopsy of metastasis: $n=52$ ). The clinical and pathological characteristics of the enrolled patients were summarized in Table S1. Of 247 patients with PM confirmed by operation (PM-confirmed), 31 cases can be detected by DRE. Based on Japanese classification of gastric carcinoma, 247 PM-confirmed patients were clarified as $\mathrm{P} 1(\mathrm{n}=69,28.0 \%), \mathrm{P} 2(\mathrm{n}=72$, $29.1 \%)$, P3( $n=106,42.9 \%)$.

\section{The performance of DRE and CT in PM patients}

The Clinicopathological characteristics of 247 cases and relationship between the true-positive and false-negative groups were listed in Table 1. By univariate and multivariable analysis, only BMI $(p=0.03)$ had relationship with the positive result of DRE (Table 2).

The sensitivities of DRE and CT for detecting PM were $12.6 \%$ (95\% CI: $7.7-17.5$ ) and $24.7 \%$ (95\% CI: 18.8-30.6). The specificities of DRE and CT were $99.8 \%$ (95\% CI: 99.7-99.9) and 97.6\% (95\% CI: 96.9-98.3). The positive predictive values (PPV) of DRE and CT for detecting PM were 91.2 (95\% CI: 84.7-97.7) and 64.9 (95\% CI: 55.5-74.3). The negative predictive values (NPV) of DRE and CT for detecting PM were 86.6 (95\% CI: 85.0-88.2) and 88.0 (95\% CI: 86.5-89.5). The positive likelihood ratio (PLR) of DRE and CT for detecting PM were 58.4 (95\% CI: 18.0-98.8) and 10.4 (95\% CI: 5.2-15.6). The negative likelihood ratio (NLR) of DRE and CT for detecting PM were 0.88 (95\% CI: 0.84-0.92) and 0.77 (95\% CI: 0.71-0.83) (Table 3). In 247 patients with PM, 14 patients were detected by CT and DRE simultaneously. 17 and 47 patients were diagnosed by DRE and CT alone (Table 4).

\section{Patients with DRE (+) may be a special subtype of stage IV GC}

To address the prognostic role of DRE $(+)$, we compared the OS of 31 DRE (+) patients with PM (PM-DRE (+)) to other 282 DRE (-) patients of stage IV (stage IV-DRE (-)). The median OS of the PM-DRE (+) and stage IV-DRE (-) groups was 10.20 (95\% CI: 9.47-10.93) and 16.97 (95\% CI: 11.00-22.93) months $(\mathrm{p}=0.03)$ (Figure 2A). The median OS of 31 PM-DRE $(+)$ patients was also lower than other 216 PM cases with DRE (-) (PM-DRE (-)). (95\% CI: 11.43-24.71, $\mathrm{P}=0.04$ ) (Figure 2B).
Table 1. The clinicopathological characteristics of 247 PM patients

\begin{tabular}{|c|c|c|c|c|c|c|}
\hline & \multicolumn{2}{|l|}{ DRE } & \multirow[b]{2}{*}{$\begin{array}{l}P \\
\text { value }\end{array}$} & \multicolumn{2}{|l|}{$\mathrm{CT}$} & \multirow[b]{2}{*}{$\begin{array}{l}P \\
\text { value }\end{array}$} \\
\hline & $\begin{array}{l}+ \\
\text { True- } \\
\text { Positive }\end{array}$ & $\begin{array}{l}\text { False- } \\
\text { negative }\end{array}$ & & $\begin{array}{l}+ \\
\text { True- } \\
\text { positive }\end{array}$ & $\begin{array}{l}\text { False- } \\
\text { negative }\end{array}$ & \\
\hline Number of patients & 31 & 216 & & 61 & 146 & \\
\hline Mean age (years) & 54.10 & 54.12 & 0.99 & 53.74 & 54.27 & 0.80 \\
\hline Gender(n) & & & 0.98 & & & 0.20 \\
\hline Female & 13 & 125 & & 30 & 74 & \\
\hline Male & 18 & 91 & & 31 & 112 & \\
\hline Mean tumor size $(\mathrm{cm})$ & 7.87 & 8.05 & 0.78 & 9.07 & 7.68 & $<0.01$ \\
\hline Tumor location (n) & & & 0.99 & & & 0.16 \\
\hline Upper $1 / 3$ & 5 & 33 & & 5 & 33 & \\
\hline Middle $1 / 3$ & 11 & 79 & & 22 & 68 & \\
\hline Lower $1 / 3$ & 15 & 104 & & 34 & 85 & \\
\hline $\begin{array}{l}\text { Signet-ring cell } \\
\text { carcinoma(n) }\end{array}$ & & & 0.57 & & & 0.81 \\
\hline No & 23 & 170 & & 47 & 146 & \\
\hline Yes & 8 & 46 & & 14 & 40 & \\
\hline \multicolumn{2}{|c|}{ Degree of peritoneal metastasis } & & $<0.01$ & & & $<0.01$ \\
\hline P1 & 0 & 69 & & 11 & 58 & \\
\hline $\mathrm{P} 2$ & 9 & 63 & & 13 & 59 & \\
\hline P3 & 22 & 84 & & 37 & 69 & \\
\hline Ascites (n) & & & 0.54 & & & $<0.01$ \\
\hline No & 16 & 135 & & 20 & 131 & \\
\hline Mild & 13 & 72 & & 31 & 54 & \\
\hline Moderate & 0 & 2 & & 1 & 1 & \\
\hline Massive & 2 & 7 & & 9 & 0 & \\
\hline \multicolumn{7}{|l|}{ Tumor marker } \\
\hline Mean CEA(ng/ml) & 14.81 & 11.48 & 0.71 & 12.49 & 11.70 & 0.91 \\
\hline Mean CA19-9(ng/ml) & 248.45 & 154.42 & 0.45 & 394.05 & 91.51 & 0.06 \\
\hline Mean CA72-4(ng/ml) & 84.42 & 29.47 & 0.08 & 66.30 & 26.55 & 0.25 \\
\hline Mean BMI (kg/m2) & 19.75 & 20.87 & 0.04 & 20.61 & 20.77 & 0.72 \\
\hline
\end{tabular}

PM: peritoneal metastasis, DRE: digital rectal examination, CT: computed tomography, BMI: body mass index

Table 2. Multivariable logistic regression analysis of associated factors for true-positive result of DRE

\begin{tabular}{llll}
\hline Variable & OR & P value & 95\% CI \\
\hline Degree of PM (P1/P2/P3) & & 0.30 & \\
Higher BMI (yes vs no) & 0.84 & 0.03 & $0.72-0.98$
\end{tabular}

PM: peritoneal metastasis, DRE: digital rectal examination, OR: odds ratio, $\mathrm{CI}$ confidence interval, BMI: body mass index

Table 3. Diagnostic results of DRE and CT

\begin{tabular}{lll}
\hline & DRE & CT \\
\hline TP & 31 & 61 \\
FP & 3 & 33 \\
TN & 1393 & 1363 \\
FN & 216 & 186 \\
p value & $<0.001$ & $<0.001$ \\
Sensitivity (\%) & 12.6 & 24.7 \\
$\mathbf{( 9 5 \% ~ C I ) ~}$ & $(7.7-17.5)$ & $(18.8-30.6)$ \\
Specificity (\%) & 99.8 & 97.6 \\
$\mathbf{( 9 5 \% ~ C I ) ~}$ & $(99.7-99.9)$ & $(96.9-98.3)$ \\
PPV (\%) & 91.2 & 64.9 \\
$\mathbf{( 9 5 \% ~ C I ) ~}$ & $(84.7-97.7)$ & $(55.5-74.3)$ \\
NPV (\%) & 86.6 & 88.0 \\
$\mathbf{( 9 5 \% ~ C I ) ~}$ & $(85.0-88.2)$ & $(86.5-89.5)$ \\
PLR & 58.4 & 10.4 \\
$\mathbf{( 9 5 \% ~ C I ) ~}$ & $(18.0-98.8)$ & $(5.2-15.6)$ \\
NLR & 0.88 & 0.77 \\
$\mathbf{( 9 5 \% ~ C I ) ~}$ & $(0.84-0.92)$ & $(0.71-0.83)$ \\
\hline TP: true-positive, FP: false-positive, TN: true-negative, FN: false-negative, PPV: \\
positive predictive value, NPV: negative predictive value, PLR: positive likelihood \\
ratios, NLR: negative likelihood ratios, DRE: digital rectal examination; CT: \\
computed tomography & \\
\end{tabular}


Table 4. Diagnostic results of DRE and CT in 247 PM patients

\begin{tabular}{llll}
\hline & \multicolumn{2}{c}{ CT } & \\
\cline { 2 - 4 } DRE & $+\boldsymbol{t}$ & - & Total \\
\hline+ & 14 & 17 & 31 \\
- & 47 & 169 & 216 \\
Total & 61 & 186 & 247 \\
\hline PM: peritoneal metastasis, DRE: digital rectal examination, CT: computed \\
tomography
\end{tabular}

In the subgroup analysis, the median OS of the P1-DRE (-) group (n=69) was 23.53 months, which was better than the PM-DRE $(+)$ group $(95 \% \mathrm{CI}$ : 13.67-33.39, $\mathrm{P}=0.01)$. The median OS of the P2-DRE (-) $(\mathrm{n}=63)$ and P3- DRE $(-)(\mathrm{n}=84)$ groups was $15.50(95 \%$ CI: 6.31-24.69) and 13.93 (95\% CI: 5.42-22.44) months. No significant differences in the OS were observed among the P2-DRE (-), P3- DRE (-) and PM-DRE (+) groups $(\mathrm{P}>0.05)$ (Figure $2 \mathrm{C}$ ).

Furthermore, we collected the data of 78 DRE $(+)$ patients without PM confirmed pathologically (nPMconfirmed). 40 of them were initial diagnosis patients without operation (initial-DRE $(+)$ ) and 38 were postoperative patients with DRE $(+)$ (post-DRE $(+)$ ). The survival of $78 \mathrm{nPM}$-confirmed DRE $(+)$ patients was significantly poorer than $247 \mathrm{PM}$-confirmed cases $(\mathrm{P}<0.001)$ (Figure 3A).

Then, we performed the subgroup analysis for DRE (+) patients in different groups. As shown in Figure 3B, no significant differences in the OS were observed between 31 PM-DRE $(+)$ patients (included in 247 cases) and $78 \mathrm{nPM}$-confirmed cases ( $\mathrm{P}>0.05)$. No significant differences in the OS were observed between the PM-DRE $(+)$ and initial-DRE $(+)$ groups $(\mathrm{P}>0.05)$ (Figure 3C). The median OS of the post-DRE $(+)$ group was 7.43 (95\% CI: 1.93-12.93) months, which was lower than the PM-DRE $(+)$ group $(\mathrm{P}=0.01)$ (Figure 3D).

\section{Construction of a nomogram to predict postoperative DRE (+)}

In another group of 1330 cases receiving radical gastrectomy, 38 patients were diagnosed as PM by
DRE postoperatively. By univariate analysis, we found age $(p<0.01)$, gender $(p=0.01)$, tumor size $(\mathrm{p}<0.01)$, quantity of ascites $(\mathrm{p}<0.01)$, perineural invasion $(p=0.03)$, extracapsular extension $(p=0.04)$, CEA $(p<0.01), T$ stage $(p<0.01)$ and $N$ stage $(p=0.03)$ were independent risk factors (Table S2). In the multivariate analysis, lower age $(<0.01)$, females $(p=0.02)$, more ascites $(p<0.01)$, and higher index of $\mathrm{CEA}(\mathrm{P}<0.01)$ were significantly associated with the increased risk of postoperative DRE $(+)$ (Table S3). Sequently, these risk factors were used to build a nomogram to predict postoperative DRE $(+)$ (Figure $4 \mathrm{~A})$, which had a bootstrap-corrected C-index of 0.73 and was well calibrated (Figure 4B).

\section{Discussion}

In this study, we determined the diagnostic and prognostic value of DRE in PM of GC for the first time. We found DRE had a higher specificity $(99.8 \%)$, PPV (91.2\%), and PLR (58.4) in diagnosis of PM compared with CT scans (specificity:97.6\%, PPV: 64.9\%, PLR: 10.4). Though the sensitivity of DRE was lower than CT scans (DRE:12.6\% CT:24.7\%), more than half of GC patients with DRE (+) could not been found by CT (17/31). These findings indicated that DRE was an effective supplement for CT and could find small metastasis easily missed by CT.

Subsequently, we found DRE (+) was a useful predictor of GC disease progression. The prognosis of DRE $(+)$ patients was much poorer than other stage IV-DRE (-) cases. This result meant that laparoscopy might be unnecessary to DRE (+) patients since DRE $(+)$ could predicate the prognosis accurately. Unnecessary laparoscopy would increase the length of hospitalization and delay onset of chemotherapy. Particularly, the patients appearing DRE (+) after radical gastrectomy represented much shorter survival [26, 27]. We speculate that exaggerated tumor burden, postoperative dystrophy and chemotherapy resistance may be related with the poor prognosis.

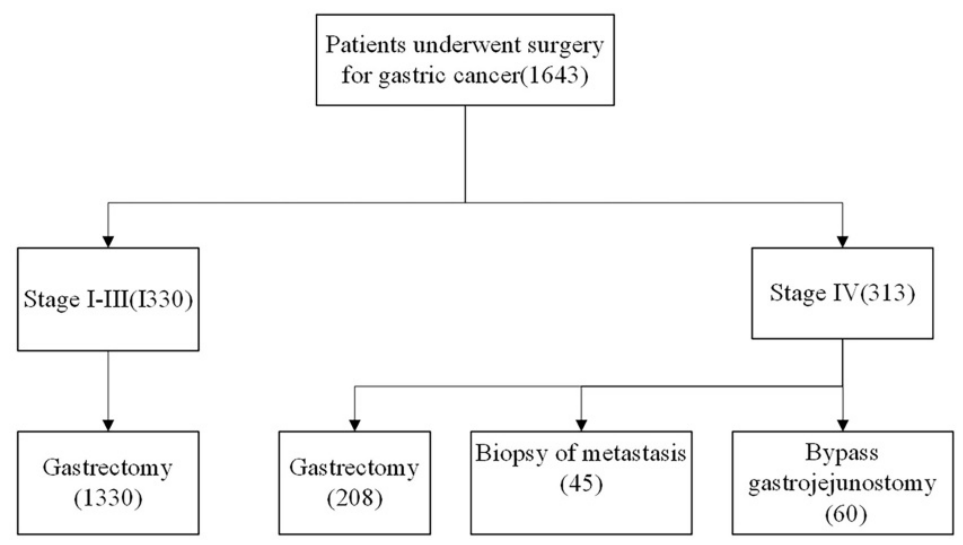

Figure 1. Consort diagram showing the study population and protocol. 

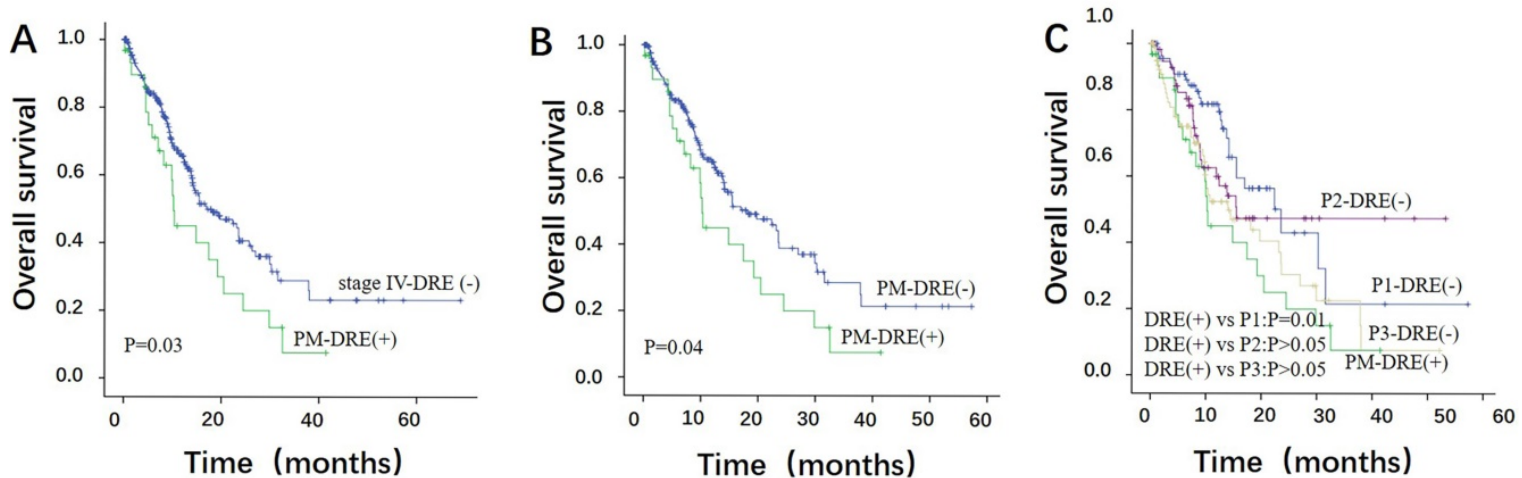

Figure 2. The overall survival (OS) of PM-DRE (+) patients. (A) A significant difference in the OS was noted between the PM-DRE (+) and stage IV-DRE (-) groups. (B) The OS of 31 PM-DRE (+) patients was lower than other 216 PM-DRE (-) patients. (C) Survival analysis of P1/P2/P3 patients with DRE (-) and PM-DRE (+) patients
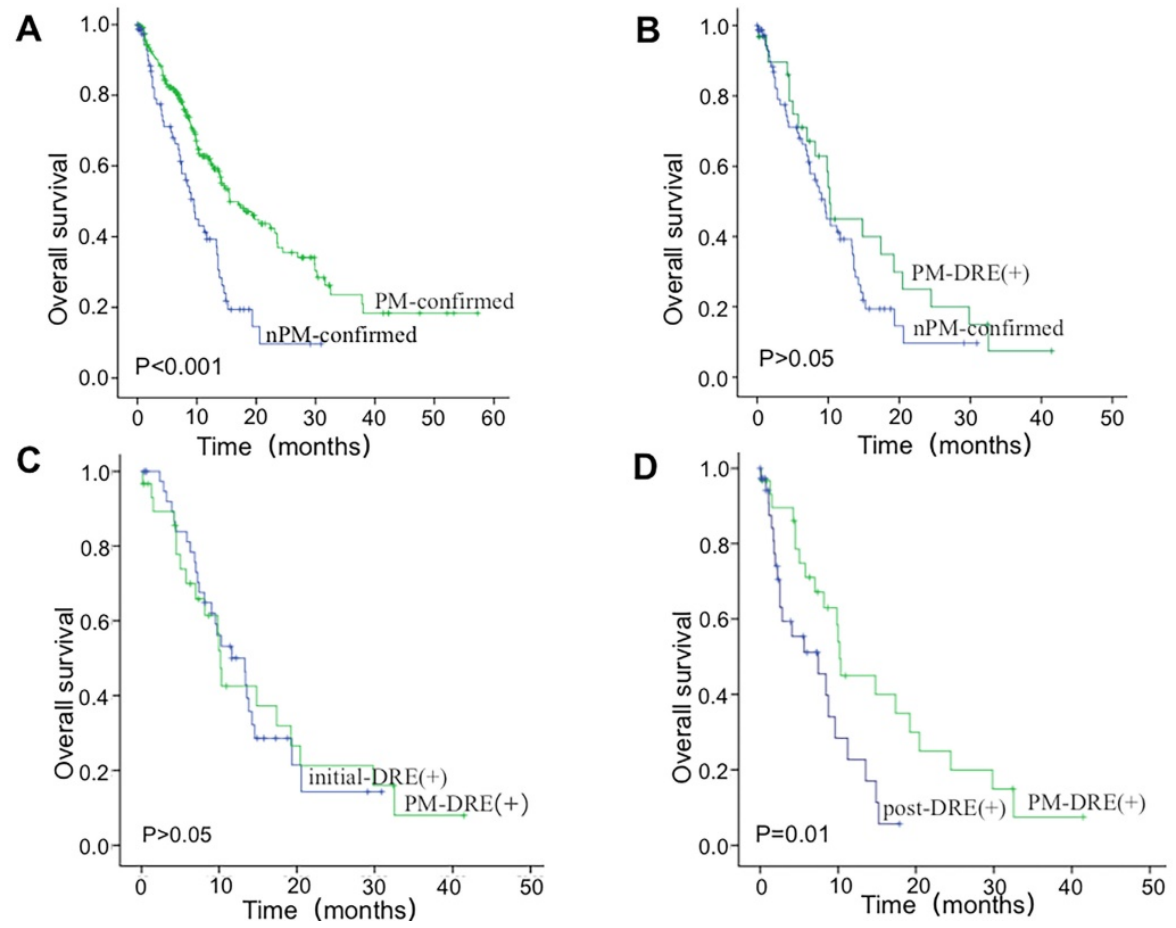

Figure 3. The overall survival (OS) of nPM-confirmed DRE (+) patients. (A) The OS of nPM-confirmed DRE $(+)$ patients was poorer than 247 cases with PM confirmed. (B) No significant difference in the OS was noted between the PM-DRE (+) and nPM-confirmed group. (C) No significant difference in the OS was noted between the PM-DRE (+) and initial-DRE (+) group. (D) The OS of the post-DRE (+) group was lower than the PM-DRE (+) group.

A

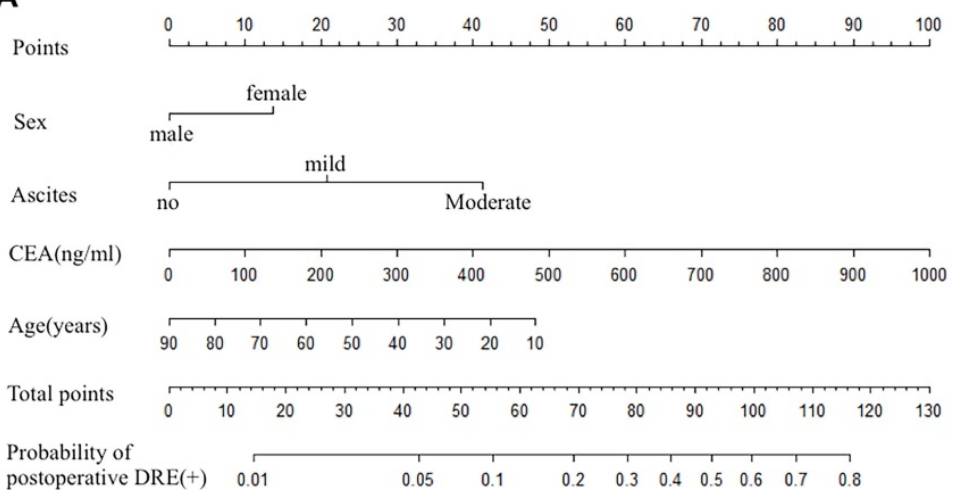

B

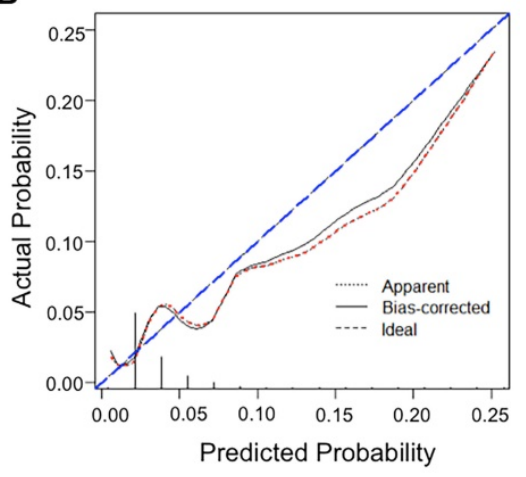

Figure 4. (A) A nomogram to predict postoperative DRE (+) for GC patients who underwent radical gastrectomy. (B) Calibration plots of the nomogram predicting. The calibration plot is a comparison between predicted and actual outcome. The blue 45-degree reference line represents an ideal model perfectly calibrated with an outcome. The red dotted line is the apparent accuracy of the nomogram, without correction for overfit. The back solid line is the bootstrap corrected performance of the nomogram, with a scatter estimate for future accuracy. 
Furthermore, age, gender, quantity of ascites, and index of CEA were confirmed as independent risk factors for DRE $(+)$ postoperatively. Similarly, Lee and Asao revealed a quantity of ascites and high index of CEA was highly sensitive in predicting PM in GC[28, 29]. Based these prognostic factors, we established a nomogram for predicting DRE (+), with a bootstrap-corrected C-index of 0.73. Previously, Rose PG et al also evaluated the clinicopathologic factors of cervical cancer and developed a nomogram for predicting pelvic recurrence[30]. The bootstrapcorrected C-index in their study was 0.73 , which was consistent with our result. Using our nomogram can predict the postoperative DRE $(+)$ easily, which help to identify asymptomatic GC patients with high-risk features and evaluate adjuvant treatment options more accurately.

Recently, chemotherapy, not unnecessary operation, has been performed for our patients with DRE (+) (data not shown). Strikingly, liver metastases, retroperitoneal lymph node metastases or malignant ascites appear soon for most DRE $(+)$ cases, which indicates that the DRE $(+)$ patients have a poor prognosis. For this subgroup of patients, supply of palliative chemotherapy and pain relief rather than invasive therapy might be a better alternative.

Currently, of 247 patients with PM confirmed, 178 were clarified as P2 and P3, which meant most patients with PM were possible be found by DRE. However, only 31 patients with PM were identified by DRE. The reasons may be related to small size of peritoneal implants, limited space of finger touch and interference of adjacent tissue. Indeed, our findings revealed that patients with high BMI were misdiagnosed more easily. Plenty of adipose tissue in pelvic cavity could interfere with the result of DRE. Marshall and Nikendei also found that the prostate could be examined by DRE completely in $91.7 \%$ of patients with a BMI below $25 \mathrm{~kg} / \mathrm{m} 2$, and in $14.3 \%$ of those with a BMI above $40 \mathrm{~kg} / \mathrm{m} 2$, which was consistent with our results[31,32]. In addition, a total of 3 false-positive DRE results were found, in which 2 were confirmed as uterine fibroids and 1 was confirmed as prostate nodular hyperplasia intraoperatively. The results indicated that the training of DRE was necessary for physicians lacking the experience.

DRE can be learned quickly with sufficient practice. However, it should be noted that DRE was often underutilized or performed in a perfunctory manner in GC patients. Recently, Wong et al. reported that DRE was decreasing in clinical practice and lots of medical students were not adequately trained to perform DRE[33]. We believe that, meticulous DRE usage and adequate training would provide accurate clinical information for GC patients with PM[33].

\section{Conclusions}

To the best of our knowledge, this study is the first to demonstrate that the diagnostic and prognostic value of DRE in GC patients with PM. DRE is an effective supplement for CT since more than half of GC patients with DRE $(+)$ could not been found by CT. Moreover, patients with DRE $(+)$ can be regarded as a special subgroup of stage IV ones with poorer prognosis. Supply of palliative care and chemotherapy might be a better alternative for them. Additionally, based on the risk factors, a nomogram established could predict postoperative DRE (+) more easily. Our Study also underscored the importance to improve awareness of and training for the DRE. As an economic, safe and convenient tool available, DRE should not become a lost art and should be utilized as a routine bedside examination tool for the evaluation of PM, especially for the advanced GC patients.

\section{Supplementary Material}

Supplementary tables.

http://www.jcancer.org/v10p1489s1.pdf

\section{Acknowledgment}

This study was supported by the Guangdong Provincial Department of Science and Technology Project (Grant No. 2016A020215089). The funder had no role in trial design, data collection, data analysis, data interpretation, or writing of the report.

\section{Author contributors}

DZX designed and managed the project. JG, YMC, ZML, YXG, WW, XWS and WL analyzed the data. JG wrote the paper. DZX revised the paper.

\section{Ethical standards}

This study has been approved by the ethics committee of Sun Yat-sen University Cancer Center and have therefore been performed in accordance with the ethical standards laid down in the 1964 Declaration of Helsinki and its later amendments.

\section{Competing Interests}

The authors have declared that no competing interest exists.

\section{References}

1. Bang YJ, Kim YW, Yang HK, Chung HC, Park YK, Lee KH, et al. Adjuvant capecitabine and oxaliplatin for gastric cancer after D2 gastrectomy (CLASSIC): a phase 3 open-label, randomised controlled trial. The Lancet. 2012; 379: 315-21.

2. Leung WK, Wu M, Kakugawa Y, Kim JJ, Yeoh K, Goh K, et al. Screening for gastric cancer in Asia: current evidence and practice. Lancet Oncology. 2008; 9: 279-87.

3. Lee K, Lee SS, Hwang JE, Jang HJ, Lee HS, Oh SC, et al. Development and Validation of a Six-Gene Recurrence Risk Score Assay for Gastric Cancer. Clinical Cancer Research. 2016; 22: 6228-35. 
4. Nashimoto A, Akazawa $\mathrm{K}$, Isobe $\mathrm{Y}$, Miyashiro I, Katai H, Kodera $\mathrm{Y}$, et al. Gastric cancer treated in 2002 in Japan: 2009 annual report of the JGCA nationwide registry. Gastric Cancer. 2013; 16: 1-27.

5. Gretschel S, Siegel R, Estevezschwarz L, Hunerbein M, Schneider U, Schlag PM. Surgical strategies for gastric cancer with synchronous peritoneal carcinomatosis. British Journal of Surgery. 2006; 93: 1530-5.

6. Association JGC. Japanese gastric cancer treatment guidelines 2014 (ver. 4). Gastric Cancer Official Journal of the International Gastric Cancer Association \& the Japanese Gastric Cancer Association. 2017; 20: 1.

7. Ajani JA, D'Amico TA, Almhanna K, Bentrem DJ, Chao J, Das P, et al. Gastric Cancer, Version 3.2016, NCCN Clinical Practice Guidelines in Oncology. Journal of the National Comprehensive Cancer Network Jnccn. 2016; 14: 1286.

8. Jackson C, Cunningham D, Oliveira J. Gastric cancer: ESMO clinical recommendations for diagnosis, treatment and follow-up. Annals of Oncology. 2009; 20 Suppl 4: 34

9. Stell DA, Carter CR, Stewart I, Anderson JR. Prospective comparison of laparoscopy, ultrasonography and computed tomography in the staging of gastric cancer. British Journal of Surgery. 1996; 83: 1260-2.

10. Ramos R, Scalon FM, Scalon MM, Dias DI. Staging laparoscopy in gastric cancer to detect peritoneal metastases: A systematic review and meta-analysis. Ejso. 2016; 42: 1315-21.

11. Kim SJ, Kim H, Kim YH, Hwang SH, Lee HS, Park DJ, et al. Peritoneal metastasis: detection with 16- or 64-detector row $\mathrm{CT}$ in patients undergoing surgery for gastric cancer. Radiology. 2009; 253: 407-15.

12. Wang C, Guo W, Zhou M, Zhu X, Ji D, Li W, et al. The Predictive and Prognostic Value of Early Metabolic Response Assessed by Positron Emission Tomography in Advanced Gastric Cancer Treated with Chemotherapy. Clinical Cancer Research. 2016; 22: 1603-10.

13. Johnstone PAS, Mcfarland JT, Riffenburgh RH, Amling CL. Efficacy of digital rectal examination after radiotherapy for prostate cancer. The Journal of Urology. 2001; 166: 1684-7.

14. Maattanen L, Auvinen A, Stenman U, Tammela TLJ, Rannikko S, Aro J, et al. Three-year results of the Finnish prostate cancer screening trial. Journal of the National Cancer Institute. 2001; 93: 552-3.

15. Soh JS, Lee HJ, Jung KW, Yoon IJ, Koo HS, Seo SY, et al. The diagnostic value of a digital rectal examination compared with high-resolution anorectal manometry in patients with chronic constipation and fecal incontinence. American Journal of Gastroenterology. 2015; 110: 1197-204.

16. Hasegawa H, Fujitani K, Nakazuru S, Hirao M, Yamamoto K, Mita E, et al. Optimal treatment change criteria for advanced gastric cancer with non-measurable peritoneal metastasis: symptom/tumor marker-based versus CT-based. Anticancer Research. 2014; 34: 5169-74.

17. Edge SBCompton CC. The American Joint Committee on Cancer: the 7th edition of the AJCC cancer staging manual and the future of TNM. Annals of Surgical Oncology. 2010; 17: 1471.

18. Marola O, Cherala R, Prusik J, Kumar V, Fama C, Wilock M, et al. BMI as a Predictor of Spinal Cord Stimulation Success in Chronic Pain Patients. Neuromodulation Technology at the Neural Interface. 2016.

19. Kenkyūkai I. Japanese classification of gastric carcinoma: Kanehara \& Co.; 1995.

20. Feng $X Y$, Wang $W$, Luo GY, Wu J, Zhou ZW, Li W, et al. Comparison of endoscopic ultrasonography and multislice spiral computed tomography for the preoperative staging of gastric cancer - results of a single institution study of 610 Chinese patients. Plos One. 2013; 8: e78846.

21. Kim HJ, Kim AY, Oh ST, Kim JS, Kim KW, Kim PN, et al. Gastric cancer staging at multi-detector row $\mathrm{CT}$ gastrography: comparison of transverse and volumetric CT scanning. Radiology. 2005; 236: 879-85.

22. Kumano $\mathrm{S}$, Murakami $\mathrm{T}$, Kim $\mathrm{T}$, Hori M, Iannaccone $\mathrm{R}$, Nakata $\mathrm{S}$, et al. T staging of gastric cancer: role of multi-detector row CT. Radiology. 2005; 237: 961.

23. Habermann CR, Weiss F, Riecken R, Honarpisheh H, Bohnacker S, Staedtler C, et al. Preoperative staging of gastric adenocarcinoma: comparison of helical CT and endoscopic US. Radiology. 2016; 230: 465-71.

24. Chang DK, Kim JW, Kim BK, Lee KL, Song CS, Han JK, et al. Clinical significance of CT-defined minimal ascites in patients with gastric cancer. World Journal of Gastroenterology. 2011; 11: 6587-92.

25. Tung TH, Chiu WH, Lin TH, Shih HC, Hsu CT. An exploration of prevalence and associated factors of nonal-coholic fatty liver disease in the Taiwanese police service. Iranian Journal of Public Health. 2011; 40: 54-62

26. Fujitani K, Yang HK, Mizusawa J, Kim YW, Terashima M, Han SU, et al. Gastrectomy plus chemotherapy versus chemotherapy alone for advanced gastric cancer with a single non-curable factor (REGATTA): a phase 3, randomised controlled trial. Lancet Oncology. 2016; 17: 309.

27. Ishigami H, Jkaisaki K. Phase II study of weekly intravenous and intraperitoneal paclitaxel combined with S-1 for advanced gastric cancer with peritoneal metastasis. Annals of Oncology. 2010; 21: 67-70.

28. Asao T, Fukuda T, Yazawa S, Nagamachi Y. Carcinoembryonic antigen levels in peritoneal washings can predict peritoneal recurrence after curative resection of gastric cancer. Cancer. 1991; 68: 44-7.

29. Lee YT, Ng EK, Hung LC, Chung SS, Ching JY, Chan WY, et al. Accuracy of endoscopic ultrasonography in diagnosing ascites and predicting peritoneal metastases in gastric cancer patients. Gut. 2005; 54: 1541-5.

30. Rose PG, Java J, Whitney CW, Stehman FB, Lanciano R, Thomas GM, et al. Nomograms Predicting Progression-Free Survival, Overall Survival, and Pelvic Recurrence in Locally Advanced Cervical Cancer Developed From an
Analysis of Identifiable Prognostic Factors in Patients From NRG Oncology/Gynecologic Oncology Group Randomized Tr. Journal of Clinical Oncology. 2015; 33: 2136-42.

31. Marshall JB. How Adequate Is Digital Rectal Exam for Prostate Cancer Screening At Colonoscopy? Can Adequacy Be Improved? Digestive Diseases \& Sciences. 2008; 53: 719.

32. Nikendei $C$, Diefenbacher $K$, Köhlhackert $N$, Lauber $H$ Huber Herrmannwerner A, et al. Digital rectal examination skills: first training experiences, the motives and attitudes of standardized patients. Bmc Medical Education. 2015; 15: 7

33. Wong RK, Drossman DA, Bharucha AE, Rao SS, Wald A, Morris CB, et al. The digital rectal examination: a multicenter survey of physicians' and students' perceptions and practice patterns. American Journal of Gastroenterology. 2012; 107: 1157-63. 